\title{
The invasion process of bovine erythrocyte by Babesia divergens: knowledge from an in vitro assay
}

Yi Sun ${ }^{1,2,3^{*}}$, Emmanuelle Moreau ${ }^{1,2,3}$, Alain Chauvin ${ }^{1,2,3}$ and Laurence Malandrin ${ }^{1,2}$

\begin{abstract}
Babesia divergens is a tick-transmitted apicomplexan parasite for which asexual multiplication in its vertebrate hosts is restricted to erythrocytes. Current knowledge of invasion of these target cells is limited. An efficient in vitro invasion assay was set up to gain access to this information. Parasites prepared from infected RBC, lysed by electroporation, and mixed with bovine RBC in a selected synthetic medium (RPMI 1640 supplemented with calcium) were able to establish subsequent cultures with parasitemia ranging from 6 to 14\%. Free parasites remaining in the invasion medium could be eliminated by Percoll gradient and culture could be pursued with the freshly invaded erythrocytes. In this way, the invasion time window could be shortened to obtain a synchronised start of the culture or to study the kinetics of invasion. With this assay we demonstrate that 1) erythrocyte invasion by $B$. divergens is a rapid process since $70 \%$ of the invasion-competent parasites invaded the RBC in less than 45 s; 2) all invasion-competent parasites achieved invasion within 10 min of contact; 3) one erythrocyte could be invaded concomitantly by two merozoites; 4) despite a synchronous start, the parasite population evolved heterogeneously resulting in a progressive loss of synchronisation. Western blot analysis of proteins collected from invasion medium were performed with sera from animals experimentally infected with $B$. divergens and highlighted several proteins. The dose-dependent, inhibitory effects of these sera on $B$. divergens invasion suggest that these proteins might be involved in the invasion process. Further investigations are required for their characterisation.
\end{abstract}

\section{Introduction}

Babesia divergens is a tick-transmitted intra-erythrocytic apicomplexan parasite, which infects cattle and a wide variety of other mammals. Experimental or natural infections by $B$. divergens have been documented in gerbils, sheep, and reindeer [1-4]. B. divergens has also been recognised, in the last 30 years, as a zoonotic agent in Europe [5-7].

Apicomplexan blood parasites differ in the range of cell types that they infect. Plasmodium spp. or Theileria spp. sporozoites first invade hepatocytes or lymphocytes, respectively, and then evolve into erythrocyte invasive merozoites [8]. In constrast, the sporozoite and the merozoite of Babesia spp., two infectious forms of the parasite, directly invade the host erythrocyte where they multiply asexually [9]. It is obvious that inhibition of

\footnotetext{
* Correspondence: yi.sun@oniris-nantes.fr

'ONIRIS, UMR1300, Biologie, Epidémiologie et Analyse de Risque en Santé Animale, Route de Gachet, La Chantrerie, BP 40706, F-44307 Nantes, France Full list of author information is available at the end of the article
}

their multiplication, and especially the step of invasion, should prevent the disease. Molecules engaged in the invasion process or contributing to its regulation are proposed as promising drug targets. In addition, since both the sporozoite and merozoite of Babesia are infectious to RBC, and since identical molecules involved in erythrocyte invasion are expressed in both stages $[10,11]$, these proteins might provide a common target for antibody-mediated inhibition of invasion.

The process of erythrocyte invasion by $B$. divergens is considered to be similar to that of Plasmodium. It is described as an initial recognition between the zoite and host cell, immediately followed by progressive internalisation at the site of merozoite apical contact, and eventually the closure of the parasitophorous vacuole [12]. Rapid invasion has been observed for Plasmodium and other Apicomplexan parasites. A recent study on P. falciparum demonstrated that merozoites recognize new target $\mathrm{RBC}$ within $1 \mathrm{~min}$ after their release from the host RBC [13]. Eighty percent of the invasion events occured

\section{Biomed Central}


within 10 min of mixing merozoites and RBC [14]. Parasite entry ensued and was complete on average $27.6 \mathrm{~s}$ after primary contact [13]. For P. knowlesi, erythrocyte invasion was shown to be accomplished within minutes after the initial contact [15]. Moreover, the penetration of Toxoplasma gondii into a vacuole formed by invagination of the plasma membrane within 25-40 s was also documented [16]. However, the cellular interactions between Babesia and its host cell have not yet been fully described, notably the disappearance of the parasitophorous vacuole, a Piroplasmidae-specific feature.

Characterisation of the molecules involved in the invasion process, as well as their corresponding erythrocyte receptors, is basic information necessary for the comprehension of RBC invasion. The proteins located on the merozoite surface, for example $\mathrm{Bd} 37$ of $B$. divergens, are usually involved in RBC adhesion [17] and are shed during the parasite internalisation process [18]. The molecules harboured in the characteristic apicomplexan secretory organelles, rhoptries, micronemes and dense granules (spherical bodies for the genus Babesia), are secreted during the invasion process by Plasmodium, Toxoplasma, and Eimeria [19-21] and are generally involved in parasite internalisation [22]. Two of these molecules have been identified in $B$. divergens, AMA-1 (Apical Membrane Antigen-1) and RAP-1 (Rhoptry Associated Protein-1). AMA-1 is secreted from the micronemes and has been characterised in hemoprotozoans in all Plasmodium species analysed [23] as well as in B. bovis [24] and B. divergens [25]. In Plasmodium, it is apparently involved in host-cell recognition, binding, and possibly motility, however its interaction with the host erythrocyte remains controversial [26]. RAP-1 has been evidenced in several Babesia species [27-30], and in B. bovis, it is supposed to be involved in erythrocyte binding and parasitophorous vacuole formation [31]. These parasitic invasion-related antigens are decapped or secreted into the surroundings during the invasion process [32]. It should therefore be possible to characterise them from the invasion medium.

In summary, the aim of our work was to set up an in vitro $B$. divergens invasion assay to characterise this process, to obtain a synchronised culture in order to focus on stage-specific activities of the parasites, and to collect the proteins in the invasion medium for further characterisation in order to elucidate the invasion mechanism.

\section{Materials and methods}

\section{In vitro culture of $B$. divergens}

$B$. divergens Rouen 87 was isolated from a human in 1987, then kept at Oniris (France), and was cultivated in vitro as described previously [33]. Briefly, parasites were cultured in bovine erythrocytes at $5 \%$ haematocrit in RPMI 1640 (Lonza, Belgium) supplemented with 20\% of heat-inactivated fetal calf serum (FCS, Lonza, Belgium). Cultures were incubated in a humidified $6 \% \mathrm{CO}_{2}$ atmosphere at $37^{\circ} \mathrm{C}$, and amplified in culture flasks to obtain the volume (up to $500 \mathrm{~mL}$ ) and parasitemia (up to 25\%) required for each experiment.

\section{Methods for preparing free parasites}

Three different methods were used to prepare live $B$. divergens parasites. First of all, free merozoites were collected from the culture medium by Percoll gradient separation. Briefly, cultures were centrifuged (2 $000 \mathrm{~g}, 5$ min) and the pellet was diluted to 1:3 with RPMI 1640. This suspension was then layered on top of a $1.08 \mathrm{~g} / \mathrm{mL}$ iso-osmotic Percoll solution prepared according to the manufacturer's instructions (Amersham Bioscience, Piscataway, USA) and centrifuged $\left(2000 \mathrm{~g}, 10 \mathrm{~min}, 20^{\circ} \mathrm{C}\right)$. The merozoites collected at the interface were washed twice in PBS (4000 g, $10 \mathrm{~min}, 4^{\circ} \mathrm{C}$ ). Besides the collection of free merozoites, the intra-erythrocytic parasites could also be liberated by RBC lysis using mechanical (high-voltage electroporation) or chemical methods (osmotic lysis). The electroporation protocol described for B. bovis was tested [32]. Parasitised cultures were centrifuged and the pellet $(2 \mathrm{~mL})$ was resuspended in an equal volume of cytomix $\left(120 \mathrm{mM} \mathrm{KCl}, 0.15 \mathrm{mM} \mathrm{CaCl}_{2}, 2 \mathrm{mM}\right.$ EGTA, $5 \mathrm{mM}$ $\mathrm{MgCl}_{2}, 10 \mathrm{mM} \mathrm{K}_{2} \mathrm{HPO}_{4} / \mathrm{KH}_{2} \mathrm{PO}_{4} \mathrm{pH}$ 8.0, 25 mM HEPES $\mathrm{pH}$ 8.0). Samples of $750 \mu \mathrm{L}$ were subjected to 5 intermittent $\left(10 \mathrm{~s}, 0^{\circ} \mathrm{C}\right)$ high-voltage pulses $(2.5 \mathrm{kV}, 200 \Omega, 25 \mu \mathrm{F})$ in a BioRad (Hercules, USA) Gene Pulser with a pulse controller using 4-mm BioRad cuvettes to lyse RBC. To eliminate the few remaining non-lysed RBC, the liberated parasites were collected by Percoll gradient and washed as described before. Osmotic lysis of the RBC was obtained by mixing a $2 \mathrm{~mL}$ pellet of infected $\mathrm{RBC}$ with $23 \mathrm{~mL}$ cytoyi (cytomix modification: $12.6 \mathrm{mM} \mathrm{K} \mathrm{HPO}_{4}, 8.74 \mathrm{mM}$ $\mathrm{KH}_{2} \mathrm{PO}_{4}, \mathrm{pH}$ 6.5) at room temperature for $5 \mathrm{~min}$, and then washing with PBS. The pelleted parasites obtained with the three methods tested, were examined on Giemsastained smears, and mixed with the invasion medium to test their viability by in vitro invasion assays.

\section{In vitro invasion media}

A synthetic medium was sought which allowed comparable invasion efficiency to that obtained with the culture medium (RPMI 1640 with 20\% FCS). Three synthetic media candidates (RPMI 1640, PBS, Tris-Sucrose) were therefore tested with or without the addition of $1 \mathrm{mM}$ calcium or $1 \mathrm{mM}$ magnesium. The culture medium was used as control.

\section{In vitro invasion assay and evaluation of invasion efficiency}

The invasion medium as well as the culture medium used as control were prepared with 5\% RBC in 24-well 
plates $(2 \mathrm{~mL} /$ well $)$ and pre-warmed at $37^{\circ} \mathrm{C}$. The pelleted parasites collected as previously described were added to each well. The efficiency of the invasion process was determined by counting the infected $\mathrm{RBC}$ on Giemsa-stained smears. Percoll separation was used to eliminate the non-invasive or dead parasites located on the surface of RBC. The effect of the Percoll separation was checked by performing an invasion assay using free merozoites collected from culture and incubated up to 120 min in RPMI 1640 with RBC. The viability of the intra-erythrocytic parasites was then controlled by washing cells collected after Percoll separation with RPMI 1640, cultivating them for $5 \mathrm{~h}$, then checking them for parasitemia. Parasitemia was calculated at each step (before, after Percoll separation, and after culture), on at least 2000 RBC.

\section{Kinetics of invasion}

The kinetics of invasion was followed in the selected synthetic medium (RPMI $1640+1 \mathrm{mM} \mathrm{CaCl}_{2}$ ), using parasites prepared by electroporation or osmotic lysis. For this purpose, RBC and parasites were mixed and harvested $0 \mathrm{~min}, 1.5 \mathrm{~min}, 3 \mathrm{~min}, 5 \mathrm{~min}, 10 \mathrm{~min}, 20 \mathrm{~min}$, $30 \mathrm{~min}, 45 \mathrm{~min}, 60 \mathrm{~min}, 120 \mathrm{~min}, 160 \mathrm{~min}$, and 240 min after initial contact. They were either observed directly on Giemsa-stained smears for morphological analysis, or centrifuged with Percoll gradient and reincubated in culture medium for parasitemia counting. Due to the speed of invasion, a rapid gradient Percoll separation was used: A layer of $500 \mu \mathrm{L}$ medium was placed on top of $500 \mu \mathrm{L}$ of Percoll in an Eppendorff tube, then centrifuged (30 s, $16000 \mathrm{~g}$ ) and washed with RPMI 1640. Each pellet was resuspended in $200 \mu \mathrm{L}$ of culture medium and transferred to a 96-well microplate for further incubation.

\section{Origin of sera used}

Seven immune sera were used to recognise the proteins collected in the supernatant during invasion. One serum was prepared from a rabbit immunised with Percoll-purified merozoites originating from a panel of $B$. divergens strains, in order to highlight as many parasitic proteins as possible. To pinpoint the proteins involved in the invasion process (i.e. those potentially secreted and thus in contact with the immune system during natural infection), we chose to use sera from two calves experimentally infected with $B$. divergens strains C139 and IV35 respectively [34] and from four sheep experimentally infected with $B$. divergens strain Rouen 87 [35]. To avoid the production of antibodies against RBC proteins, the calves and sheep were infected with parasites cultivated in vitro in their own erythrocytes.

\section{Analysis of proteins contained in the invasion test supernatant (SDS-PAGE electrophoresis and Western blotting)}

The conditions of efficient invasion were determined from the above assays. In summary, the merozoites were prepared by high-voltage electroporation, then incubated with $5 \% \mathrm{RBC}$ in RPMI $1640+1 \mathrm{mM} \mathrm{CaCl} 2$ for $10 \mathrm{~min}$. After incubation, the culture was centrifuged $\left(16000 \mathrm{~g}, 10 \mathrm{~min}, 4^{\circ} \mathrm{C}\right)$ to eliminate the $\mathrm{RBC}$ and remaining free parasites. After addition of Protease Inhibitor Mix 1:100 (Amersham Bioscience), the supernatant was concentrated 12 times by Centrifugal Filter Devices (Millipore-Amicon ultra-15, Billerica, USA) (10 $000 \mathrm{~g}, 2 \mathrm{~h}, 4^{\circ} \mathrm{C}$ ) with a $10 \mathrm{kDa}$ cut-off. Potential contamination with erythrocyte proteins derived from the preparation of parasites for invasion was determined by electroporating non-infected bovine erythrocytes under the same conditions. The resulting pellet was added to the invasion medium, then the supernatant was collected and concentrated in the same way as described above.

The concentrated supernatants were separated on a 12\% SDS-PAGE gel, stained with Coomassie Brilliant Blue R-250 or transferred to a nitrocellulose membrane, which was blocked with $10 \%$ skimmed milk in TBS/0.1\% Tween 20 (TBSt) overnight. After each incubation, all washes were done three times for $5 \mathrm{~min}$ in TBSt. The blots were incubated for $1 \mathrm{~h}$ in sera from immunised rabbits, or experimentally-infected calves or sheep, diluted $1 / 100$ in TBSt with 5\% skimmed milk. After washing, the membranes were incubated with anti-IgG (anti-rabbit IgG; anti-bovine IgG or anti-sheep IgG coupled to alkaline phosphatase, Sigma Aldrich, Milwaukee, USA) diluted $1 / 1000$ in TBSt with $5 \%$ skimmed milk for $30 \mathrm{~min}$. The colorimetric reaction was carried out for 5 min with BCIP/NBT liquide substrate system (Sigma Aldrich).

\section{Inhibition of invasion with anti-sera}

One calf serum (C139) and one sheep serum (S-M-SC) were then selected on the basis of the WB analysis and used to validate our protocol in an invasion inhibition assay and to determine the potential function of the proteins detected during invasion. Different dilutions (1/ $2,1 / 4,1 / 8)$ of the antisera were added separately to the invasion medium, before the addition of live merozoites prepared by electroporation. Their effects on invasion were compared to those of the same dilutions of sera from non-infected calves or sheep. After incubation for $10 \mathrm{~min}$, the RBC were washed in RPMI 1640, then reincubated in culture medium for $5 \mathrm{~h}$ before parasitemia control. 


\section{Statistical analysis}

A non-parametric test was used for the statistical analysis. Wilcoxon was used for the comparison between two elements, and Kruskal Wallis was used for comparison of more than two elements. The test was considered "statistically significant" when the p-value was less than 0.05 .

\section{Results}

Percoll separation allows elimination of the extraerythrocytic parasites and the calculation of invasion efficiency

Parasitemia was measured before and after Percoll separation, and after $5 \mathrm{~h}$ incubation in culture medium to determine the efficiency of Percoll separation in eliminating parasites that did not invade erythrocytes during the invasion test and to control the non-toxicity of Percoll on intra-erythrocytic parasites. These measurements were performed 5, 10 and 120 min after the initial contact between RBC and merozoites. Regardless of the time of contact, a significant decrease in parasitemia from 14 fold $(5 \mathrm{~min})$ to 4 fold $(120 \mathrm{~min})$, was obtained after Percoll separation (Figure 1). The observed decrease in parasitemia according to the time of contact before Percoll separation was not correlated with a corresponding increase of invading parasites (after Percoll). No significant differences in parasitemia were observed

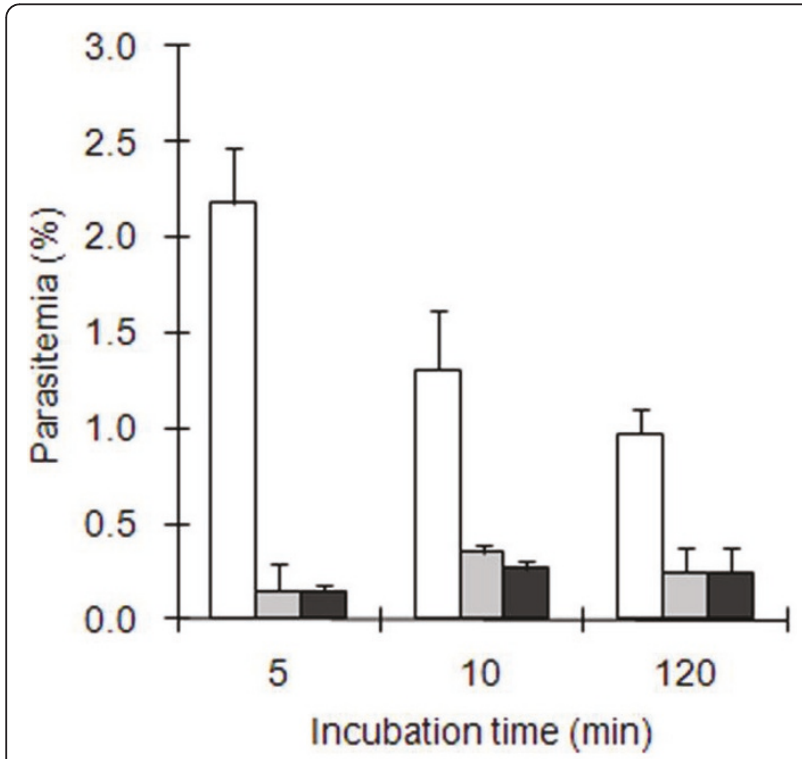

Figure 1 Improvement of invasion efficiency evaluation Merozoites collected from culture supernatant were used to perform the invasion assay in RPMI 1640. Parasitemia was counted before (white bars), after Percoll separation (grey bars) and after $5 \mathrm{~h}$ incubation in culture medium (black bars). Each figure represents the average value of the triplicate assays and error bars indicate the standard deviation. before or after additional incubation in culture medium at any of the three contact times (Figure 1). That is to say that Percoll is compatible with the subsequent in vitro growth of $B$. divergens.

\section{Erythrocyte invasion is more efficient in RPMI 1640 supplemented with $\mathrm{Ca}^{2+}$}

In order to choose a suitable synthetic medium for performing the invasion test, we compared the invasion efficiency in culture medium (RPMI $1640+20 \%$ FCS) with the invasion efficiencies obtained using three synthetic media (PBS, Tris-Sucrose and RPMI 1640). Calcium and magnesium were added to each synthetic medium to find out if they could improve invasion efficiency. Free parasites were obtained by RBC osmotic lysis, and a $30 \mathrm{~min}$ incubation time was used. Among the three synthetic media tested, the same invasion efficiency as the culture medium was only obtained with RPMI 1640 (Figure 2). Parasitemia was significantly lower than the control when PBS or Tris-Sucrose was used. Adding calcium or magnesium had no significant effect except for RPMI 1640 supplemented with calcium. Therefore RPMI $1640+1 \mathrm{mM} \mathrm{CaCl}_{2}$ was selected for the in vitro invasion assays.

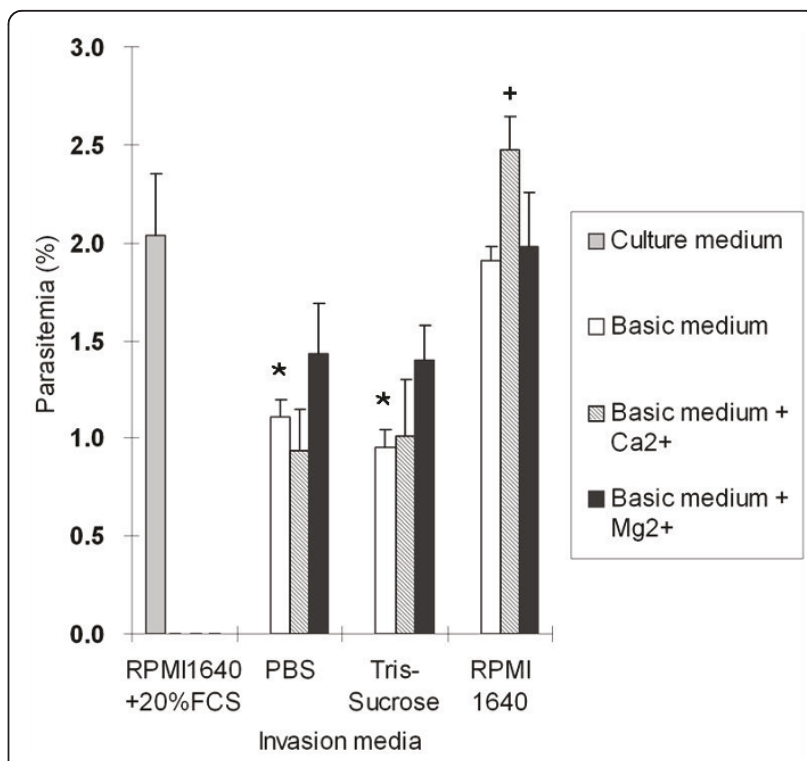

Figure 2 Influence of medium composition on erythrocyte invasion efficiency. The invasion assays were performed in 3 basic media (white bars), and compared to the complete medium (gray bar). $\mathrm{CaCl}_{2}$ (striped bars) or $\mathrm{MgCl}_{2}$ (black bars) were added at a concentration of $1 \mathrm{mM}$ into the basic media separately. Each figure represents the average value of parasitemia from the triplicate assays and error bars indicate the standard deviation. Significant differences $(P<0.05)$ between complete medium and basic media are indicated with stars, while significant differences due to addition of calcium of magnesium in each basic medium are indicated with crosses. 
Free, viable, and invasion-competent parasites were obtained by high-voltage electroporation

Starting with the same culture material, RBC osmotic lysis and RBC electroporation produced 5 times and 29 times more "invasion-competent" parasites respectively than merozoites from the culture supernatant (Figure 3). Similar results were obtained in a second assay (data not shown).

B. divergens invasion of the erythrocyte is a rapid process $B$. divergens parasites prepared by RBC lysis (osmotic and electroporation) were used to follow the kinetics of invasion by analysing parasitemia after contact with RBC ranging from 0 to $240 \mathrm{~min}$. Although a huge difference in the maximum parasitemia attained in these two assays was observed (which confirmed the choice of electroporation as the better method of parasite preparation), the trends of the two curves were comparable (Figure 4a). B. divergens invasion of $\mathrm{RBC}$ occurred within seconds. A contact of 45 $\mathrm{s}$ (the time required to mix $\mathrm{RBC}$ with parasites, and to perform Percoll separation) was indeed sufficient to attain $50 \%$ (RBC osmotic lysis) and 70\% (RBC electroporation) of maximum parasitemia. Ten minutes after the initial contact, all "invasion-competent" parasites had penetrated the $\mathrm{RBC}$, since no further increase in parasitemia was observed over time. Microphotographs of Giemsa-stained smears prepared immediately after contact (about $5 \mathrm{~s}$ ), at

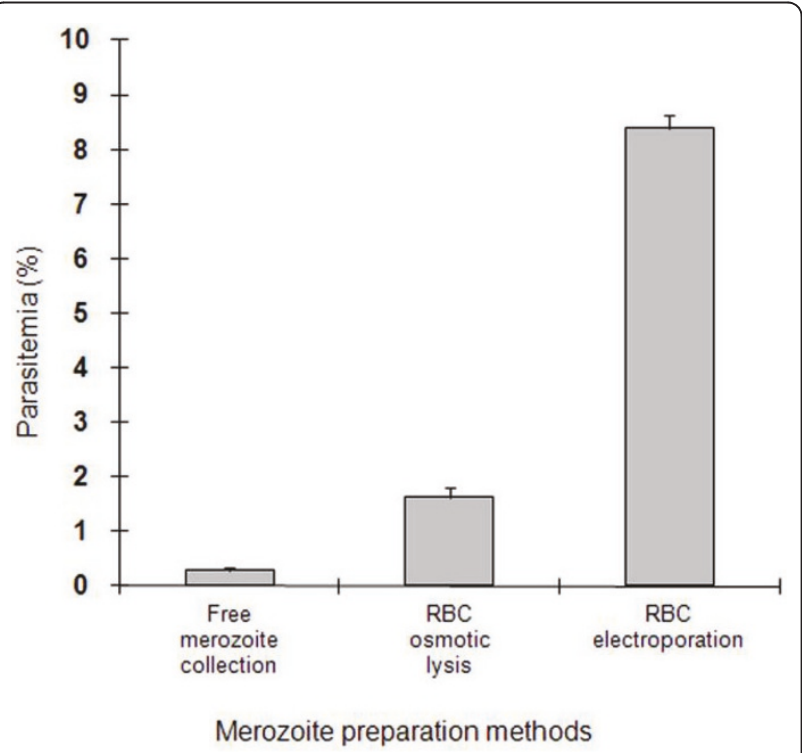

Figure 3 Influence of the methods of merozoite preparation on erythrocyte invasion efficiency. Parasites from the culture medium were collected by three different methods. The invasion assays were performed in RPMI $1640+1 \mathrm{mM} \mathrm{CaCl}_{2}$. The experiment was performed twice with similar results, and one of them is presented here. Each figure represents the average value of the triplicate assays and error bars indicate the standard deviation.
$10 \mathrm{~min}$ and $120 \mathrm{~min}$ after contact and before Percoll separation were taken to show the changes in parasite morphology (Figure 4b). Free parasites, some adhering to the $\mathrm{RBC}$ surface, were visible after contact $(0,10 \mathrm{~min})$. Their quantities seemed to diminish with time (120 min). Intra-cellular parasites were visible immediately after the contact with RBC. Their shape changed rapidly, from condensed intra-erythrocytic black particles at the very beginning, to trophozoites with the appearance of cytoplasms (white areas) inside the parasite at ten minutes, which expanded to become clearly visible at $120 \mathrm{~min}$. Parasites with homogeneous size and shape were still visible 120 min after the beginning of the culture.

\section{The intra-erythrocytic development of $B$. divergens is asynchronous}

The development of $B$. divergens after invasion was followed by calculating at different incubation times the percentage of parasites at the following developmental stages: trophozoite, beginning and end of cellular division (Table 1). To improve synchronisation, late invasions were avoided by eliminating extra-erythrocytic parasites $10 \mathrm{~min}$ after the contact between RBC and parasites. Up to $3 \mathrm{~h}$ after RBC invasion, the only parasite forms observed in the culture were trophozoites (Table 1). The onset of division was observed at $4 \mathrm{~h}$. The proportion of the population at this stage increased for at least $2 \mathrm{~h}$, peaked around $6 \mathrm{~h}$ after invasion and then decreased. Fully divided parasites were observed $1 \mathrm{~h}$ after the onset of division, and accounted for about one third of the observed parasite population $9 \mathrm{~h}$ after invasion. The trophozoite population exhibited a considerable decline after invasion, from $6 \mathrm{~h}$ on, but remained the main population detected. Eight hours after invasion, about $30 \%$ of the parasites (approximate sum of parasite percentages seen at the end of division from 5 to $8 \mathrm{~h}$ ) had fully divided to produce about $8 \times$ $10^{7}$ merozoites $/ \mathrm{mL}$, each invading a new $\mathrm{RBC}$ and resulting in a new trophozoite.

\section{RBC can be invaded by two $B$. divergens merozoites}

Occasionally, after an invasion assay, two trophozoites were observed within the same RBC (Figure 5) and accounted for about 3\% of the infected RBC. Their occurrence was observed just after invasion. They were similar in shape and size not only within a double infected erythrocyte, but also compared to single troprozoites, and this was observed $1 \mathrm{~h}$ and $4 \mathrm{~h}$ after invasion (Figure 5). Their presence in such an assay indicates the ability of two merozoites to invade a single target cell.

\section{B. divergens antigens were released into the environment during the invasion assay}

The proteins collected in the supernatant were analysed by SDS-PAGE. As a result of RBC lysis, many 


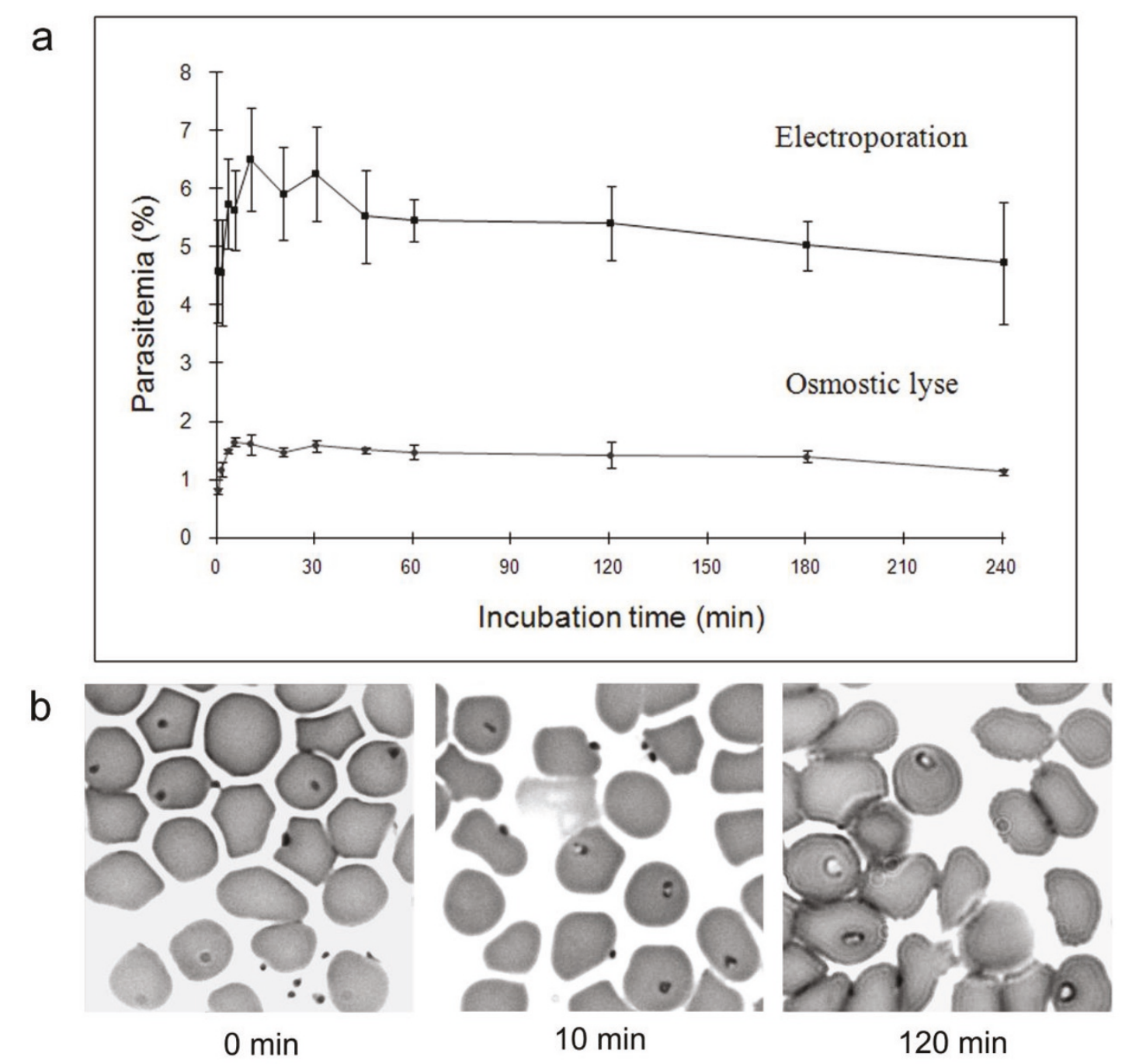

Figure 4 a). Kinetics of erythrocyte invasion. Curves indicate the percentage of erythrocytes invaded with parasites prepared by highvoltage electroporation (squares) or by osmotic lysis (rounds) according to the time of incubation. Each figure represents the average value of parasitemia from the triplicate assays and error bars indicate the standard deviation. b). Photomicrographs of bovine erythrocytes following an invasion assay with merozoites prepared by electroporation. The photos were taken immediately after contact and at 10 min and $120 \mathrm{~min}$.

contaminating RBC proteins were present in the control lane (lane S) as well as in the test lane (lane P) (Figure 6a). The main parasite-derived proteins collected during the invasion assay (molecular weights ranging from 36 $\mathrm{kDa}$ to $104 \mathrm{kDa}$ ) are highlighted with arrows on lane P.

The collected proteins were also analysed by Western blotting with different $B$. divergens antisera. Many contaminating $\mathrm{RBC}$ proteins were recognised by rabbit antiserum, thereby confirming the presence of $R B C$ contamination in the parasite preparation used for immunisation (Figure 6b). As expected, few (Figure 6d) or none (Figure $6 \mathrm{c}$ ) of these proteins were detected in sera obtained from experimentally-infected animals.
Table 1 Percentage of $B$. divergens parasites at the stages of trophozoite, onset of division and end of cellular division following an invasion assay.

\begin{tabular}{cccc}
\hline & Trophozoite (\%) & Onset of division (\%) & End of division (\%) \\
\hline $3 \mathrm{~h}$ & 100 & 0 & 0 \\
\hline $4 \mathrm{~h}$ & 98.7 & 1.3 & 0 \\
\hline $5 \mathrm{~h}$ & 96.6 & 1.5 & 1.9 \\
\hline $6 \mathrm{~h}$ & 88.8 & 6.0 & 5.2 \\
\hline $8 \mathrm{~h}$ & 75.7 & 2.6 & 21.7 \\
\hline $9 \mathrm{~h}$ & 65.6 & 1.9 & 32.5
\end{tabular}

Parasites at each stages were counted from 3 to $9 \mathrm{~h}$ after the contact between free parasites and red blood cells. 


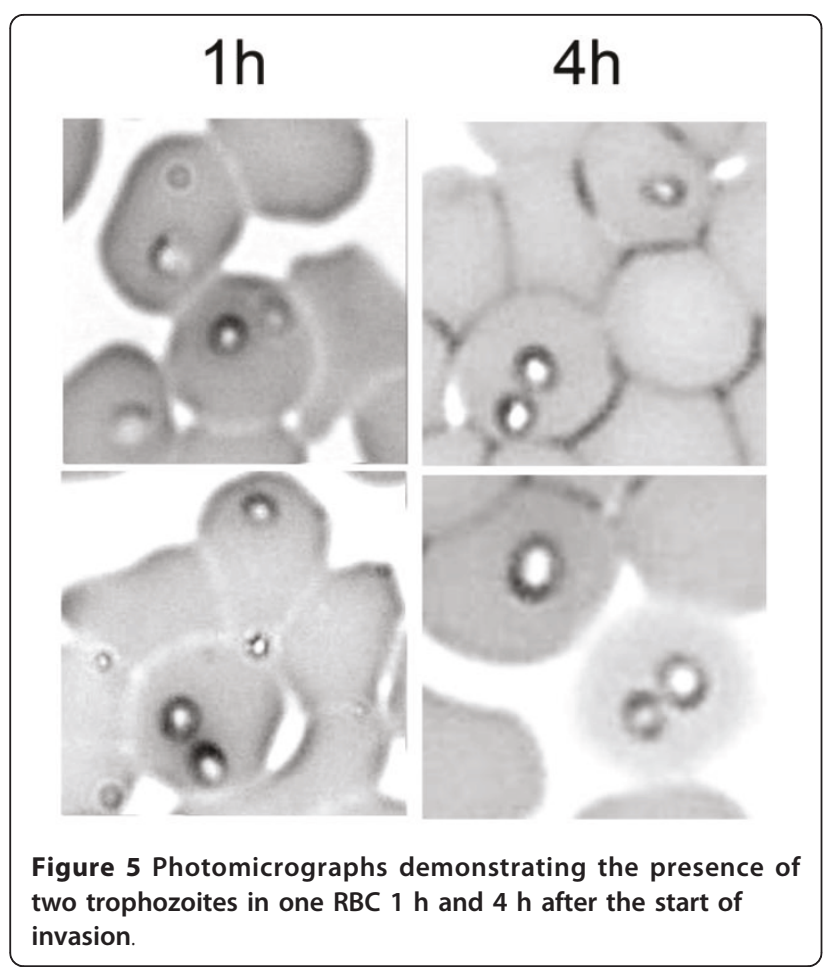

Three proteins with molecular weights of about 110 $\mathrm{kDa}, 102 \mathrm{kDa}$, and $95 \mathrm{kDa}$ (respectively No. 1, 2, 3 on Figure 4) were recognised by all tested sera (7 sera in total, 3 of them shown as representative), even by calf sera collected at an early stage of infection (14 days) (Figure $6 \mathrm{c}$ ). The other antigens were detected in different combinations depending on the serum.

\section{Antisera from experimentally-infected animals inhibit $B$. divergens invasion}

The invasion assay was used to test the inhibitory effect of two selected sera on invasion (Figure 7). For both sera, an inhibition of invasion was observed at each dilution tested (up to 1/8) and the effect was dose-dependent. Invasion was reduced by up to $80 \%$ in the case of calf antiserum. Sheep negative serum also had an inhibitory effect on invasion, an effect that had already been noted on growth of $B$. divergens [36]. It could therefore be postulated that the proteins detected by Western blotting with these sera had a role in the invasion process.

\section{Discussion}

Studies of the invasion process in Babesia are of pivotal importance since only this Apicomplexan genus has infectious forms (merozoites and sporozoites) which start their host invasion directly by erythrocyte penetration. However, very little information is available on this process compared, for example, to that of Plasmodium. We focussed our studies on $B$. divergens because it is the major hemoparasite of cattle in Europe with zoonotic transmission. We developed an in vitro invasion

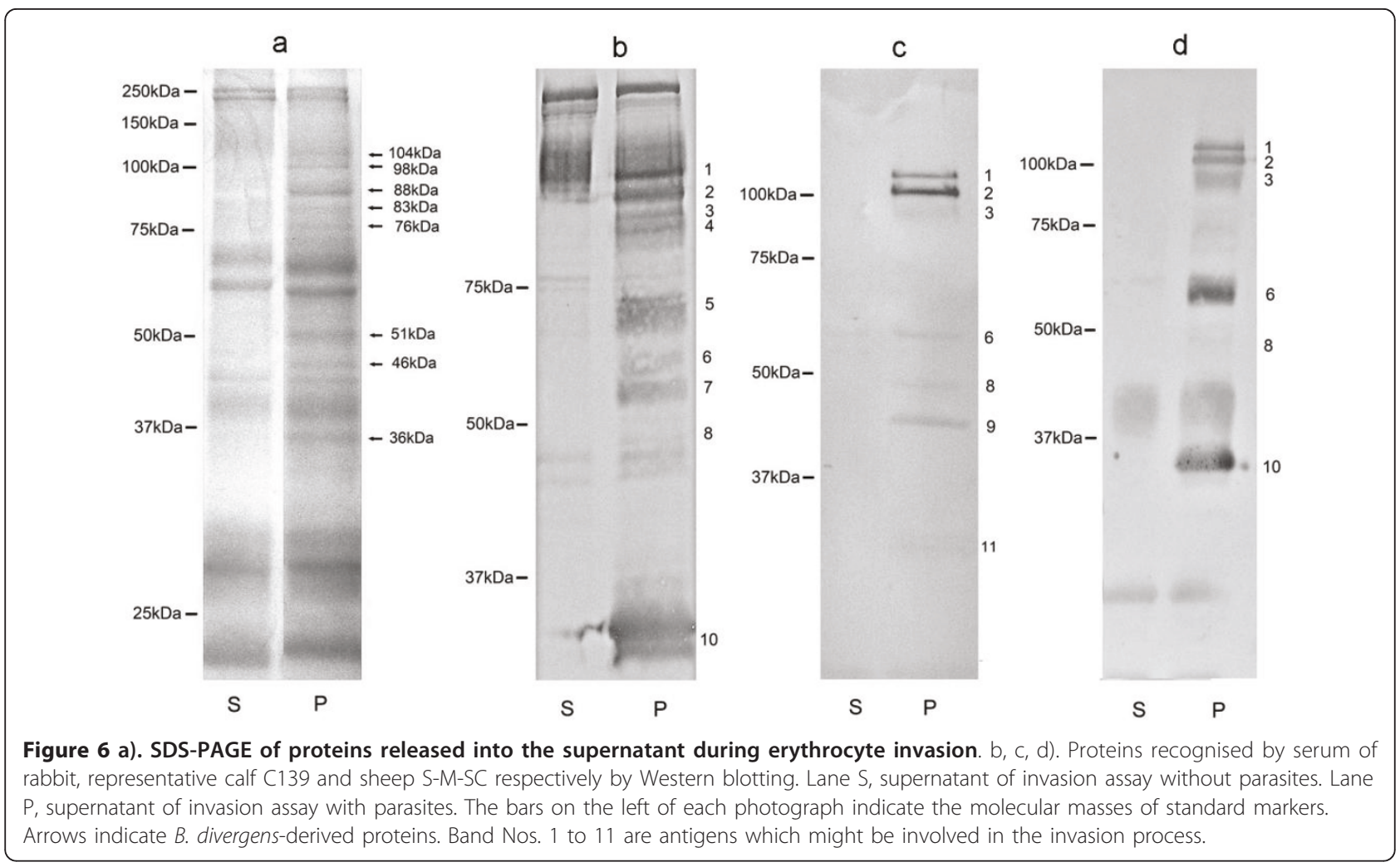



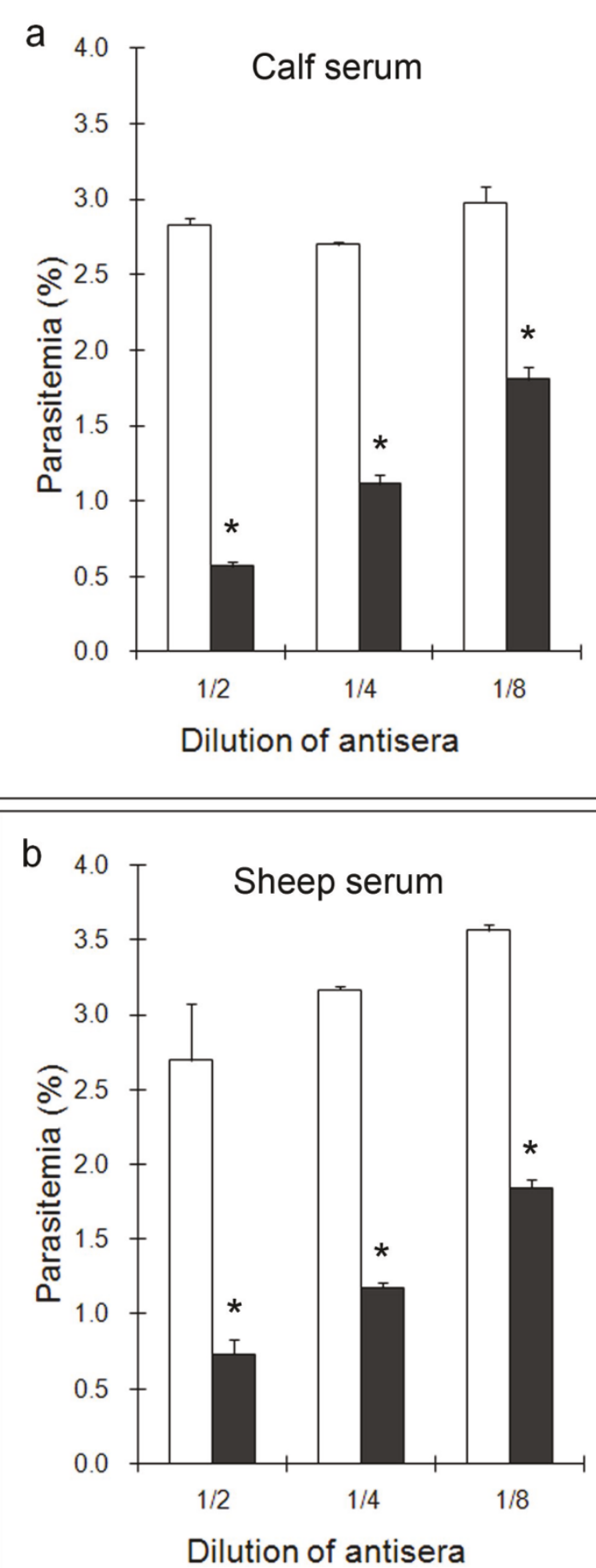

Figure 7 Inhibition of invasion by sera directed against $B$. divergens. Invasion was performed with antisera of calf C139 and sheep S-M-SC (black bars) and compared to the negative sera of calf and sheep (white bars). Each figure represents the average value of the triplicate assays and error bars indicate the standard deviation. Significant differences $(P<0.05)$ between antisera and negative sera are indicated with stars. assay to acquire knowledge of the process and molecules involved in invasion of the RBC by $B$. divergens.

The choice of medium used to obtain efficient invasion in vitro is crucial. Of the three media tested, RPMI $1640+20 \%$ FCS and RPMI 1640 exhibited the same invasion efficiency. Although serum is known to be essential for Babesia growth in vitro, we demonstrate here, as reported for B. bovis and P. falciparum [14,32], that it is not necessary for erythrocyte invasion by $B$. divergens. The invasion efficiency measured with PBS (used for the B. bovis invasion test in Franssen et al., [32]) and Tris-Sucrose was significantly lower. The relative invasion efficiencies with PBS compared to the control medium were comparable for B. bovis (about 60\%) and $B$. divergens (54\%). However, the positive effect of calcium addition to PBS was significant only in the case of B. bovis invasion. A positive and significant effect of calcium addition was observed in our study only when RPMI 1640 was used (parasitemia increased by 30\%). The role of extra-cellular calcium in merozoite invasion of the erythrocyte is well documented for P. falciparum [37] and B. bovis [32]. In our case, its effect depended on the medium.

Compared to the two other methods of merozoite preparation (collection of free merozoites in the culture supernatant and liberation of intra-cellular merozoites by osmotic lysis), electroporation of infected RBC was able to provide a greater number of "invasion-competent" parasites. It is extremely difficult to estimate the number of free and alive parasites due to their size and aggregation. Moreover, parasites might remain in the erythrocyte ghosts after lysis and be unable to invade a new RBC. The best way to compare the quality and quantity of "invasion-competent" parasites is to perform an in vitro invasion assay. Thus, a Percoll separation and reincubation in culture medium were performed to guarantee the reliability of the estimated invasion efficiency. Due to the death of extra-erythrocytic parasites, a large number of them were actually free or adhering to the surface of RBC but not internalized, and were therefore efficiently removed by the Percoll separation. Indeed, the parasitemia count obtained after incubation in culture medium is more reliable due to the size of the parasite.

Electroporation is a simple and effective method for the disruption of cell membranes by application of an external electric field. We confirmed the suitability of this method for $B$. divergens, as already described for $B$. bovis [32]. The amount of "invasion-competent" merozoites produced is large enough to establish a culture with parasitemia ranging from 5 to $14 \%$, a range of parasitemia occurring during the acute phases of bovine Babesiosis. Such high parasitemia could not be achieved by osmotic lysis which repeatedly produced subsequent 
cultures with parasitemia ranging between 1 and $2 \%$. The harmful effect of the medium used for RBC lysis was probably responsible for the death of the released parasites. A maximum parasitemia of $0.5 \%$ was obtained when free merozoites collected from the culture supernatant were used. This probably reflects the limited number of free merozoites in the supernatant or their low viability. According to our data, we collected about $5 \times 10^{6}$ "invasion-competent" merozoites from $40 \mathrm{~mL}$ of culture, calculated from the parasitemia after invasion (Figure 1). Other authors were able to collect about $10^{8}$ merozoites from the same volume of culture [25]. There are two possible explanations for this difference. Firstly, the maximum parasitemia values of the initial cultures were different ( $20 \%$ vs. $70 \%$, due to the erythrocyte type, bovine vs. human), which would influence the amount of free merozoites. Secondly, only about $10 \%$ of the collected merozoites were demonstrated to be "invasioncompetent" (Figure 1), a characteristic that was not investigated by the authors. As demonstrated in our study, $B$. divergens invasion is an extremely rapid process meaning that "invasion-competent" merozoites remain freely in the medium for a very short time. The merozoites collected from the supernatant were probably those that had remained for a long time in the medium and were thus probably "non-invasion-competent" (dead or non-viable).

By electroporation, $6.4 \times 10^{9}$ infected $\mathrm{RBC}$ were necessary to obtain a culture with $6 \%$ parasitemia. This corresponds to $4.8 \times 10^{7}$ infected RBC after invasion, that is to say that only $0.75 \%$ of the prepared parasites were "invasion-competent", i.e. viable merozoites. This low percentage might be explained by the asynchronous status of the culture. The invasion yield could be optimized by using a synchronized culture to increase the proportion of geminate-stage parasites.

The in vitro invasion assay presented in this study permits the analysis of the kinetics of invasion, especially its rapidity. As observed for other Apicomplexan parasites, the invasion of $B$. divergens proceeded rapidly after the initial contact between parasites and RBC, since $70 \%$ of the "invasion-competent" parasites invaded a RBC within $45 \mathrm{~s}$, and the maxium level of invasion was attained within $10 \mathrm{~min}$. This result corresponded to the observation of $B$. bovis erythrocyte invasion that the attachment to $\mathrm{RBC}$ was achieved within $5 \mathrm{~min}$, whereas $41 \%$ of the maximum level of invasion was attained after $15 \mathrm{~min}$, and the maximum after $1 \mathrm{~h}$ [32]. During the asexual multiplication of intra-erythrocytic Apicomplexans, the merozoite is the only stage in contact with the host immune system. B. divergens and other Apicomplexan parasites have developed an efficient invasion mechanism to minimise the time of contact and ensure survival in their vertebrate hosts.
Another application of this in vitro invasion assay is the collection of proteins released by the parasites during the invasion process. Host-cell invasion by apicomplexan parasites involves the successive exocytosis of secretory organelles into the RBC and environment [38]. These proteins, present in in vitro culture, can induce a high degree of protection against virulent heterologous challenge exposure $[39,40]$. They are thought to be potential sources of antigens to induce protective immunity. Moreover, their characterisation should provide a better understanding of the molecular support underlying host cell invasion.

A minimum invasion level has to be reached to ensure that the amount of proteins is large enough for their characterisation, which is why an efficient method of merozoite preparation is crucial to the invasion assay. With our assay we were able to collect, in $1 \mathrm{~mL}$ of invasion medium, the proteins secreted by $4.8 \times 10^{7}$ to 1.1 $\times 10^{8}$ invasion competent parasites within $10 \mathrm{~min}$, depending on the assay (with parasitemia of subsequent cultures ranging from 6 to 14\%).

Proteins collected from the invasion assay supernatant were labelled by Western blotting with different antisera. As expected, the antibodies of rabbit antisera prepared by immunisation with Percoll-separated merozoites recognised numerous proteins including probably house-keeping ones. On the contrary, sera from experimentally-infected animals (calves or sheep) recognised fewer proteins (about 6). These sera are most probably enriched in antibodies against the most antigenic proteins, and among them, merozoite surface antigens or proteins secreted during invasion by the apical organelles of the merozoite (rhoptries and micronemes) could be included. Among these proteins, some were recognised by calf sera collected early during the infection process (proteins No.1, 2, 3, Figure 6c). Others (proteins No. 8 and 9, Figure 6c) could correspond to already described invasion-related proteins, such as AMA-1 ( 48 kDa, after the precursor cleavages) [25] and RAP-1 ( 46 kDa) [29]. An invasion inhibition assay was performed to determine the participation of these recognised proteins in the invasion process. The significant and dose-dependent inhibitory effect of sera from infected animals on erythrocyte invasion suggests such a role. Further investigations are needed for their identification as potential vaccine candidates.

The asexual multiplication of $B$. divergens, in vivo as well as in vitro, is asynchronous and methods of obtaining a synchronised Babesia culture are lacking. Research using synchronised culture is essential to study DNA transcription and protein expression at different parasitic stages. Synchronised cultures could also be used to identify the precise cellular steps (DNA replication, cell division) targeted by drugs. B. bovis in vitro invasion 
with isolated parasites was not able to provide a synchronised culture [32]. For the purpose of obtaining a synchronised culture of $B$. divergens, non-lysed RBC (infected or not) were eliminated after electroporation. Moreover, the invasion window time was restricted to $10 \mathrm{~min}$, and could be further reduced by removing extra-erythrocytic parasites from the RBC earlier to obtain an improved synchronised start. Even so, the intra-erythrocytic development of the parasite did not remain synchronised. Parasites at the stage of onset of division were detected from $4 \mathrm{~h}$ on and still at $9 \mathrm{~h}$ after invasion included, thus indicating the extremely heterogeneous intra-cellular development of this parasite. This results in the mix of the trophozoites from the first generation and that of the second generation, which explained the maintenance of a trophozoite population in the culture, even when the time window for invasion to occur was limited to $10 \mathrm{~min}$. However, this assay could be used to study gene transcription and expression in relation to the onset of main cell-cycle features, such as DNA replication or cell division events. Synchronisation is well developed for Plasmodium [41-45], Toxoplasma [46] and Theileria [47], but was barely achieved for Babesia [32]. A synchronised culture of $P$. falciparum with a large age range (3-5 h) was obtained by density gradients $[41,42]$, differential osmotic lysis of parasitised erythrocytes [43], temperature shifts [44], and recently optimised to a specific cell-cycle phase with growth inhibitors [45]. Further improvement of the synchronisation of $B$. divergens cultures will probably rely on drugs that specifically block key points in the cell cycle. However, the effects of such molecules on $B$. divergens remain uncharacterised.

We established an efficient in vitro invasion assay for $B$. divergens. Thanks to this assay, we demonstrated that RBC invasion occurred in less than $45 \mathrm{~s}$, and that the invasion of one erythrocyte by two merozoites is possible. Synchronisation, even partial, of a culture could allow transcription and translation studies during the cell-cycle. By uncoupling invasion and intra-erythrocytic development, studies of the actions of inhibitory compounds that specifically target these stages would be feasible. The presence in the assay medium of proteins involved in invasion is suspected, since inhibition of invasion with antibodies from experimentally-infected animals was obtained. The invasion assay provides the possibility of more accurate and specific comparisons of the invasion process between $B$. divergens and other Apicomplexa. More specifically, Babesia-specific characteristics such as the disappearance of the parasitophorous vacuole or more generally the molecular basis of host specificity could be investigated.

\section{Acknowledgements}

This work was supported by research funds from the French National Institute for Agricultural Research, and Oniris (France). Y. Sun's PhD thesis is financed by Région Pays de Loire. We wish to acknowledge the group IECM for allowing access to the electroporation apparatus and S. Nusinovici for his help with the statistical analysis.

\section{Author details}

'ONIRIS, UMR1300, Biologie, Epidémiologie et Analyse de Risque en Santé Animale, Route de Gachet, La Chantrerie, BP 40706, F-44307 Nantes, France. ${ }^{2}$ INRA Angers-Nantes, BP 71627, F-44307 Nantes, France. ${ }^{3}$ Université Nantes Angers Le Mans, France.

\section{Authors' contributions}

YS carried out most of the experimental part with the help of LM for the invasion tests. YS and LM wrote the manuscript. AC was YS PhD supervisor and revised the manuscript. EM and LM participated in the design, coordination and revision of the study. All authors read and approved the final manuscript.

\section{Competing interests}

The authors declare that they have no competing interests.

Received: 26 October 2010 Accepted: 11 May 2011

Published: 11 May 2011

\section{References}

Chauvin A, Valentin A, Malandrin L, L'Hostis M (2002) Sheep as a new experimental host for Babesia divergens. Vet Res 33:429-433. doi:10.1051/ vetres:2002029.

Langton C, Gray JS, Waters PF, Holman PJ (2003) Naturally acquired babesiosis in a reindeer (Rangifer tarandus tarandus) herd in Great Britain. Parasitol Res 89:194-198. doi:10.1645/0022-3395(2003)089[0194:MEOCOB]2.0.CO;2.

Lewis D, Williams H (1979) Infection of the Mongolian gerbil with the cattle piroplasm Babesia divergens. Nature 278:170-171. doi:10.1038/278170a0.

Nilsson O, Nordkvist M, Ryden L (1965) Experimental Babesia divergens infection in reindeer (Rangifer tarandus). Acta Vet Scand 6:353-359

Gray JS (2006) Identity of the causal agents of human babesiosis in Europe. Int J Med Microbiol 296(Suppl 40):131-136

Homer MJ, Aguilar-Delfin I, Telford SR, Krause PJ, Persing DH (2000) Babesiosis. Clin Microbiol Rev 13:451-469. doi:10.1128/CMR.13.3.451-469.2000.

Zintl A, Mulcahy G, Skerrett HE, Taylor SM, Gray JS (2003) Babesia divergens, a bovine blood parasite of veterinary and zoonotic importance. Clin Microbiol Rev 16:622-636. doi:10.1128/CMR.16.4.622-636.2003.

Shaw MK (1997) The same but different: the biology of Theileria sporozoite entry into bovine cells. Int J Parasitol 27:457-474. doi:10.1016/S0020-7519(97)000155 .

Mehlhorn H, Schein E (1984) The piroplasms: life cycle and sexual stages. Adv Parasitol 23:37-103

Mosqueda J, McElwain TF, Stiller D, Palmer GH (2002) Babesia bovis merozoite surface antigen 1 and rhoptry-associated protein 1 are expressed in sporozoites, and specific antibodies inhibit sporozoite attachment to erythrocytes. Infect Immun 70:1599-603. doi:10.1128/IAI.70.3.1599-1603.2002.

Mosqueda J, McElwain TF, Palmer GH (2002) Babesia bovis merozoite surface antigen 2 proteins are expressed on the merozoite and sporozoite surface, and specific antibodies inhibit attachment and invasion of erythrocytes. Infect Immun 70:6448-55. doi:10.1128/IAI.70.11.6448-6455.2002.

Preiser P, Kaviratne M, Khan S, Bannister L, Jarra W (2000) The apical organelles of malaria merozoites: host cell selection, invasion, host immunity and immune evasion. Microbes Infect 2:1461-1477. doi:10.1016/S1286-4579(00) 01301-0.

Gilson PR, Crabb BS (2009) Morphology and kinetics of the three distinct phases of red blood cell invasion by Plasmodium falciparum merozoites. Int J Parasitol 39:91-96. doi:10.1016/i.ijpara.2008.09.007.

Boyle MJ, Wilson DW, Richards JS, Riglar DT, Tetteh KK, Conway DJ, Ralph SA, Baum J, Beeson JG (2010) Isolation of viable Plasmodium falciparum merozoites to define erythrocyte invasion events and advance vaccine and drug development. Proc Natl Acad Sci USA 107:14378-14383. doi:10.1073/ pnas.1009198107. 
Miller LH, Aikawa M, Dvorak JA (1975) Malaria (Plasmodium knowlesi) merozoites: immunity and the surface coat. J Immunol 114:1237-1242

Morisaki JH, Heuser JE, Sibley LD (1995) Invasion of Toxoplasma gondii occurs by active penetration of the host cell. J Cell Sci 108:2457-2464

Delbecq S, Auguin D, Yang YS, Lohr F, Arold S, Schetters T, Precigout E, Gorenflot A, Roumestand C (2008) The solution structure of the adhesion protein $\mathrm{Bd} 37$ from Babesia divergens reveals structural homology with eukaryotic proteins involved in membrane trafficking. J Mol Biol 375:409-424. doi:10.1016/j.jmb.2007.08.019.

Delbeca S, Precigout E, Vallet A, Carcy B, Schetters TP, Gorenflot A (2002) Babesia divergens: cloning and biochemical characterization of $\mathrm{Bd} 37$. Parasitology 125:305-312

Camus D, Hadley TJ (1985) A Plasmodium falciparum antigen that binds to host erythrocytes and merozoites. Science 230:553-556. doi:10.1126/ science.3901257.

Carruthers VB, Sibley LD (1997) Sequential protein secretion from three distinct organelles of Toxoplasma gondii accompanies invasion of human fibroblasts. Eur J Cell Biol 73:114-123

Tomley FM, Bumstead JM, Billington KJ, Dunn PP (1996) Molecular cloning and characterization of a novel acidic microneme protein (Etmic-2) from the apicomplexan protozoan parasite, Eimeria tenella. Mol Biochem Parasitol 79:195-206. doi:10.1016/0166-6851(96)02662-X.

Yokoyama N, Okamura M, Igarashi I (2006) Erythrocyte invasion by Babesia parasites: current advances in the elucidation of the molecular interactions between the protozoan ligands and host receptors in the invasion stage. Vet Parasitol 138:22-32. doi:10.1016/j.vetpar.2006.01.037.

Triglia T, Healer J, Caruana SR, Hodder AN, Anders RF, Crabb BS, Cowman AF (2000) Apical membrane antigen 1 plays a central role in erythrocyte invasion by Plasmodium species. Mol Microbiol 38:706-718. doi:10.1046/ j.1365-2958.2000.02175.x

Gaffar FR, Yatsuda AP, Franssen FF, de Vries E (2004) Erythrocyte invasion by Babesia bovis merozoites is inhibited by polyclonal antisera directed against peptides derived from a homologue of Plasmodium falciparum apical membrane antigen 1. Infect Immun 72:2947-2955. doi:10.1128/IAl.72.5.29472955.2004.

Montero E, Rodriguez M, Oksov Y, Lobo CA (2009) Babesia divergens apical membrane antigen 1 and its interaction with the human red blood cell. Infect Immun 77:4783-4793. doi:10.1128/AAl.00969-08.

Mitchell GH, Thomas AW, Margos G, Dluzewski AR, Bannister LH (2004) Apical membrane antigen 1, a major malaria vaccine candidate, mediates the close attachment of invasive merozoites to host red blood cells. Infect Immun 72:154-158. doi:10.1128/IAI.72.1.154-158.2004.

Dalrymple BP, Casu RE, Peters JM, Dimmock CM, Gale KR, Boese R, Wright IG (1993) Characterisation of a family of multi-copy genes encoding rhoptry protein homologues in Babesia bovis, Babesia ovis and Babesia canis. Mol Biochem Parasitol 57:181-192. doi:10.1016/0166-6851(93)90194-3.

Kappmeyer LS, Perryman LE, Hines SA, Baszler TV, Katz JB, Hennager SG, Knowles DP (1999) Detection of equine antibodies to Babesia caballi by recombinant $B$. caballi rhoptry-associated protein 1 in a competitiveinhibition enzyme-linked immunosorbent assay. J Clin Microbiol 37:2285-2290

Skuce PJ, Mallon TR, Taylor SM (1996) Molecular cloning of a putative rhoptry associated protein homologue from Babesia divergens. Mol Biochem Parasitol 77:99-102. doi:10.1016/0166-6851(96)02570-4.

Suarez CE, Palmer GH, Hotzel I, McElwain TF (1998) Structure, sequence, and transcriptional analysis of the Babesia bovis rap-1 multigene locus. Mol Biochem Parasitol 93:215-224. doi:10.1016/50166-6851(98)00032-2.

Yokoyama N, Suthisak B, Hirata H, Matsuo T, Inoue N, Sugimoto C, Igarashi I (2002) Cellular localization of Babesia bovis merozoite rhoptry-associated protein 1 and its erythrocyte-binding activity. Infect Immun 70:5822-5826. doi:10.1128/IAl.70.10.5822-5826.2002.

Franssen FF, Gaffar FR, Yatsuda AP, de Vries E (2003) Characterisation of erythrocyte invasion by Babesia bovis merozoites efficiently released from their host cell after high-voltage pulsing. Microbes Infect 5:365-372. doi:10.1016/S1286-4579(03)00041-8.

Malandrin L, L'Hostis M, Chauvin A (2004) Isolation of Babesia divergens from carrier cattle blood using in vitro culture. Vet Res 35:131-139. doi:10.1051/ vetres:2003047.

Malandrin L, Jouglin M, Sun Y, Brisseau N, Chauvin A (2010) Redescription of Babesia capreoli (Enigk and Friedhoff, 1962) from roe deer (Capreolus capreo/us): isolation, cultivation, host specificity, molecular characterisation and differentiation from Babesia divergens. Int J Parasitol 40:277-284. doi:10.1016/j.jpara.2009.08.008

Moreau E, Jouglin M, Chauvin A, Malandrin L (2009) Babesia divergens experimental infection of spleen-intact sheep results in long-lasting parasitemia despite a strong humoral response: preliminary results. Vet Parasitol 166:205-211. doi:10.1016/j.vetpar.2009.08.021.

Malandrin L, Jouglin M, Moreau E, Chauvin A (2009) Individual heterogeneity in erythrocyte susceptibility to Babesia divergens is a critical factor for the outcome of experimental spleenintact sheep infections. Vet Res 40:25. doi:10.1051/vetres/2009008.

McCallum-Deighton N, Holder AA (1992) The role of calcium in the invasion of human erythrocytes by Plasmodium falciparum. Mol Biochem Parasitol 50:317-323. doi:10.1016/0166-6851(92)90229-D.

Dubremetz JF, Garcia-Reguet N, Conseil V, Fourmaux MN (1998) Apical organelles and host-cell invasion by Apicomplexa. Int J Parasitol 28:1007-1013. doi:10.1016/50020-7519(98)00076-9.

Patarroyo JH, Prates AA, Tavares CA, Mafra CL, Vargas Ml (1995) Exoantigens of an attenuated strain of Babesia bovis used as a vaccine against bovine babesiosis. Vet Parasitol 59:189-199. doi:10.1016/0304-4017(94)00756-3.

Valentin A, Precigout E, L'Hostis M, Carcy B, Gorenflot A, Schrevel J (1993) Cellular and humoral immune responses induced in cattle by vaccination with Babesia divergens culture-derived exoantigens correlate with protection. Infect Immun 61:734-741

Jensen JB (1978) Concentration from continuous culture of erythrocytes infected with trophozoites and schizonts of Plasmodium falciparum. Am J Trop Med Hyg 27:1274-1276

Kutner S, Breuer WV, Ginsburg H, Aley SB, Cabantchik ZI (1985) Characterization of permeation pathways in the plasma membrane of human erythrocytes infected with early stages of Plasmodium falciparum: association with parasite development. J Cell Physiol 125:521-527. doi:10.1002/jcp.1041250323.

Lambros C, Vanderberg JP (1979) Synchronization of Plasmodium falciparum erythrocytic stages in culture. J Parasitol 65:418-420. doi:10.2307/3280287.

Rojas MO, Wasserman M (1993) Effect of low temperature on the in vitro growth of Plasmodium falciparum. J Eukaryot Microbiol 40:149-152. doi:10.1111/ j.1550-7408.1993.tb04895.x.

Naughton JA, Bell A (2007) Studies on cell-cycle synchronization in the asexual erythrocytic stages of Plasmodium falciparum. Parasitology 134:331-337. doi:10.1017/S0031182006001466.

Conde de Felipe MM, Lehmann MM, Jerome ME, White MW (2008) Inhibition of Toxoplasma gondii growth by pyrrolidine dithiocarbamate is cell cycle specific and leads to population synchronization. Mol Biochem Parasitol 157:22-31. doi:10.1016/.j.molbiopara.2007.09.003.

Stagg DA, Chasey D, Young AS, Morzaria SP, Dolan TT (1980) Synchronization of the division of Theileria macroschizonts and their mammalian host cells. Ann Trop Med Parasitol 74:263-265

doi:10.1186/1297-9716-42-62

Cite this article as: Sun et al.: The invasion process of bovine erythrocyte by Babesia divergens: knowledge from an in vitro assay. Veterinary Research 2011 42:62.

\section{Submit your next manuscript to BioMed Central and take full advantage of:}

- Convenient online submission

- Thorough peer review

- No space constraints or color figure charges

- Immediate publication on acceptance

- Inclusion in PubMed, CAS, Scopus and Google Scholar

- Research which is freely available for redistribution

Submit your manuscript at www.biomedcentral.com/submit
C Biomed Central 